\title{
LA EDUCACIÓN EN CIENCIA, TECNOLOGÍA Y SOCIEDAD PARA LA FORMACIÓN INVESTIGATIVA DEL INGENIERO CIVIL.
}

\author{
Marlene Vilar de los Santos Finalé \\ https://orcid.org/0000-0001-6739-1673 \\ Ramón Vidal Pla López ${ }^{2}$ \\ https://orcid.org/0000-0003-4773-360X
}

\section{RESUMEN}

El objetivo de este artículo es explicar un proceder didáctico para contribuir a la formación investigativa de los estudiantes desde el proceso de enseñanza aprendizaje (PEA) de la asignatura Problemas Sociales de la Ciencia y la Tecnología (PSCT), en la carrera de Ingeniería Civil de la Universidad Máximo Gómez Báez, Cuba. Se parte de analizar las insuficiencias que persisten en la formación de los ingenieros civiles respecto a su desarrollo investigativo causado por las características que asume el proceso de formación. A partir del método de estudio de contenidos de autores como: Restrepo Gómez (2003), Hilarraza (2012), Zamora Vega (2014), Márquez Valdés y Acosta Urbano (2014), entre otros, se establece la relación entre Formación Investigativa - Enfoque Ciencia Tecnología y Sociedad (CTS) - Proceso de Enseñanza Aprendizaje de la asignatura PSCT. Se aplicó una encuesta a profesores para determinar las causas de las insuficiencias del proceso de formación de los estudiantes. El estudio de contenido y el diagnóstico realizado permitió elaborar un proceder didáctico para perfeccionar el proceso de enseñanza aprendizaje de la referida asignatura. El nuevo proceder didáctico se implementó en el tercer año de la carrera de Ingeniería Civil de la Universidad Máximo Gómez Báez, Cuba. Se realizaron evaluaciones sistemáticas de los indicadores establecidos y se obtuvieron resultados significativos registrados y sometidos a pruebas estadísticas. Se demuestran coincidencias de los resultados con los fundamentos teóricos estudiados a través de diferentes autores.

Palabras Clave: Enfoque CTS; Formación Investigativa; Problemas Sociales de la Ciencia y Tecnología; Proceso de enseñanza aprendizaje.

\section{A educaşão em Ciência, Tecnologia e Sociedade para a formação investigativa do engenheiro civil. \\ RESUMO}

O objectivo deste artigo é explicar um procedimento didáctico para contribuir na formação investigativa dos estudantes a partir do processo de ensino-aprendizagem (PEA) da disciplina Problemas Sociais da Ciência e Tecnologia (PSCT), no curso de Engenharia Civil da Universidade Máximo Gómez Báez, Cuba. Parte-se da análise das insuficiências que persistem na formação dos engenheiros civis em relação ao seu desenvolvimento investigativo causado pelas características que assumem o processo de formação. A partir do método de estudo de conteúdos de autores como: Restrepo Gómez (2003), Hilarraza (2012), Zamora Vega (2014), Márquez Valdés e Acosta Urbano (2014) entre outros, estabelece-se a relação entre Formação Investigativa - Enfoque Ciência Tecnologia e Sociedade (CTS) - Processo de Ensino - Aprendizagem da disciplina PSCT. Foi aplicado um questionário a professores, para determinar as causas das insuficiências do processo de formação dos estudantes. $O$ estudo de conteúdos e o diagnóstico realizado permitiu elaborar um procedimento didáctico para aperfeiçoar o processo de ensino aprendizagem da referida disciplina. O novo procedimento didáctico foi implementado no terceiro ano do curso de Engenharia Civil da Universidade Máximo Gómez Báez, Cuba. Realizaram-se avaliações sistemáticas dos indicadores estabelecidos e foram obtidos resultados significativos registados e submetidos a provas estatísticas. Foram demonstradas coincidências dos resultados com os fundamentos teóricos estudados através de diferentes autores. Palavras-Chave: Enfoque CTS; Formação Investigativa; Problemas Sociais da Ciência e Tecnologia; Proceso de ensino-aprendizagem.

\footnotetext{
1 Profesora Auxiliar de la Universidad Máximo Gómez Báez, Cuba. Email: marlene@sma.unica.cu
}

2 Profesor titular de la Universidad Máximo Gómez Báez, Cuba. Email rplalopez@sma.unica.cu 
Education in Science, Technology and Society for the investigative training of the civil engineer.

\section{ABSTRACT}

The objective of this article is to explain a didactic procedure to contribute to the research training of students from the teaching-learning process (PEA) of the subject Social Problems of Science and Technology (PSCT), in the Civil Engineering career of the University Máximo Gómez Báez, Cuba. It starts from analyzing the insufficiencies that persist in the training of civil engineers regarding their research development caused by the characteristics assumed by the training process. From the content study method of different authors Restrepo Gómez (2003), Hilarraza (2012), Zamora Vega (2014), Márquez Valdés y Acosta Urbano (2014), among others, the relationship between Investigative Training - Science Technology and Society Approach (CTS) - Teaching-Learning Process of the PSCT subject is established. A survey was applied to teachers to determine causes of the insufficiencies of the student training process. The content study and the diagnosis made allowed the elaboration of a didactic procedure to improve the teaching learning process of the subject. The new didactic procedure was implemented in the third year of the Civil Engineering career of the University Máximo Gómez Báez, Cuba. Systematic evaluations of established indicators and significant results were recorded and subjected to statistical tests. Theorists studied through different authors.

Keywords: CTS approach; Investigative Training; Social Problems of Science and Technology; Teaching-learning process.

\section{Introducción}

La formación de profesionales en la Educación Superior se orienta al egreso de ciudadanos actos para las exigencias sociales del siglo XXI, dueños de una cultura universal con la identidad y autenticidad de su comunidad y país. Sujetos comprometidos con la historia y las tradiciones de su entorno, profundamente reflexivos, esencialmente humanos en sus convicciones y comportamientos sociales, con capacidad transformadora, indagativa y argumentativa, potencialmente preparados para los desempeños laborales y profesionales; con posibilidades para insertarse en el vertiginoso avance de la ciencia, la tecnología, el arte y dispuestos a crecer tanto en el orden de la preparación técnica y profesional como social y espiritual, lo que se resume en profesionales capaces, comprometidos, flexibles y trascendentes.

Por tal razón, la Educación Superior tiene el desafío de realizar un proceso de formación profesional que afiance un modelo educativo que sea productivo, creativo e innovador, que se contraponga al informativo, estimule la participación activa de formados y formadores, vinculados con las novedades de la producción y los servicios en función del desarrollo de competencias que los revelen como creadores y modificadores, que se preparan durante la vida.

En consonancia con ello la universidad cubana contemporánea forma profesionales a partir de un modelo de amplio perfil cuya cualidad esencial es la profunda formación básica, sustentada en el dominio de los aspectos cruciales el desempeño profesional, que garantiza la capacidad para el éxito en distintas esferas de la actividad laboral. Estos aspectos hacen que se le reconozca como universidad científica, tecnológica y humanista (Núñez-Jover, 2019).

Autores como: Álvarez de Zayas (1990); Márquez-Valdés y Acosta-Bandomo (2014); ZamoraVera (2014) y Pla-López (2017) han enriquecido con sus estudios y aportes el proceso de formación de los profesionales y el proceso de investigación científica y han contribuido al perfeccionamiento sistemático de la práctica pedagógica y científico investigativa en la Educación Superior. Sin embargo, no se ha llegado a integrar un sistema categorial revelador de la dinámica del proceso de formación investigativa.

Otros autores han indagado en el enfoque CTS en el ámbito educativo, Acevedo-Díaz (1997); Osorio (2002); Vilches, Gil, Cañal de León (2010), para perfeccionar el PEA de los PSCT.

Márquez-Valdés y Acosta-Bandomo (2014) abordan la relación del enfoque CTS con la formación de la competencia investigativa de los estudiantes de la carrera Cultura Física y precisan que se caracteriza por tener un carácter crítico en relación con la visión esencialista y grupalista de la ciencia y la tecnología, una perspectiva interdisciplinaria integradora de disciplinas como Filosofía de la Ciencia, Historia de la Ciencia y la Tecnología, la Sociología del conocimiento científico, la Teoría de la Educación y la Economía del cambio tecnológico, un carácter contextual que interrelaciona a la ciencia y la tecnología para la configuración de valores en la vida de una sociedad 
determinada, poseer una naturaleza multifactorial que promueve el análisis y solución de problemas científico-tecnológicos sobre la base de la comunión de factores: políticos, ambientales, éticos, económicos, históricos, educacionales, entre otros, evidenciar un espíritu formacional de la responsabilidad con la ciencia y tecnología que propicia una acción social y una esencia democrática que involucra en la búsqueda de soluciones a problemas científico-tecnológicos.

Los autores referenciados ofrecen una visión de la ciencia que tiene influencia en la formación de los estudiantes desde el punto de vista investigativo, consideran que esta sirve de fundamento a los estudios CTS y coinciden en que la ciencia y la tecnología tienen naturaleza social pues se desarrollan en este tipo de actividad de los seres humanos. Ciencia tecnología para ellos son: fenómenos socialmente significativos, pues influyen en la vida social y cultural y están determinadas por dicha sociedad. Son resultado de un proceso de trabajo colectivo realizado en instituciones sociales, empresas, universidades, etcétera. Los consideran como fenómenos sociales a los que le son inherentes la ideología de la sociedad en que se desarrollan. Creen que tienen potencialidades ilimitadas para resolver los problemas del mundo actual si forman parte de estrategias integradoras de totalidad social, que deben ser objeto de un control social. La ciencia y la tecnología deben definir los valores y los fines sociales, sus funciones sociales tienen un carácter histórico-concreto y para ello adoptan atributos nacionales, regionales, etcétera. La alfabetización científico-tecnológica es un problema de primer orden, y debe llegar a todos los niveles educacionales.

El acercamiento de los autores antes citados a establecer relación entre enfoque CTS y la formación de competencias investigativas es el antecedente más cercano a este estudio. Sin embargo, no se han encontrado otros que indaguen sobre la manera que esta relación puede contribuir a la formación de los estudiantes de ingeniería desde el punto de vista investigativo, lo cual tiene sus particularidades.

A pesar de los avances en la ciencia pedagógica acerca de la formación profesional e investigativa de los estudiantes, aún existen dificultades que los limitan en su desempeño, que se expresan en: insuficiencias en la búsqueda de información científica para resolver los problemas, en el análisis crítico de información, en la identificación de problemas, en el planteamiento de soluciones a problemas y en la socialización de resultados de los procesos investigativos.

Las carreras deben replantear su proceso de formación profesional y ofrecer a los estudiantes posibilidades para desarrollar competencias que permitan identificar y solucionar problemas e integrar saberes con significado. Se necesita cambiar la actitud práctica orientada con el propósito de fomentar una actitud indagativa, propiciar la investigación, crear cultura investigativa desde la propia dinámica de ejecución del proceso de formación profesional, dirigida al manejo de herramientas básicas de investigación, a la identificación y formulación adecuada de problemas, que el individuo debe convertir en oportunidades, accediendo a la información con eficacia y estando en posibilidad de transformarla en conocimiento.

Para formar un profesional competente se necesita contar con un sistema que desarrolle determinadas habilidades y cualidades, las que no distan de la concepción del modelo educativo actual, no obstante, demanda modificaciones en su dinámica.

Las insuficiencias observadas en la formación de los estudiantes universitarios desde el punto de vista investigativo, que contradicen el ideal de profesional que se necesita, deben ser abordadas desde la investigación científica. La experiencia en el ejercicio de esta profesión, condujeron a los investigadores a observar cómo se comportaba esa situación en el PEA de la asignatura PSCT en el tercer año de la carrera Ingeniería Civil de las Universidad de Ciego de Ávila para perfeccionarlo en función de contribuir a la formación de los estudiantes desde el punto de vista investigativo.

El objetivo de este artículo es explicar el proceder didáctico para contribuir a la formación investigativa de los estudiantes desde el proceso de enseñanza aprendizaje de la asignatura Problemas Sociales de la Ciencia y la Tecnología, en la carrera de Ingeniería Civil. 
Los resultados que se exponen en este artículo se insertan en un proyecto de investigación de la Universidad de Ciego de Ávila, Cuba titulado "El desarrollo del pensamiento científico de los estudiantes y sus implicaciones axiológicas"

\section{Metodología el proceso investigativo}

Para desarrollar el proceso investigativo se utilizó una metodología general con un enfoque sistémico que privilegia la dialéctica de lo cuantitativo y lo cualitativo apoyada en variados métodos para la obtención de cada uno de los resultados, tales como: el método analítico sintético, el inductivo deductivo, el histórico lógico, el estudio de contenido, a partir de diferentes autores que permitió sistematizar los principales fundamentos del proceso investigativo.

Se realizó un estudio de contenidos de 13 artículos, seleccionados a partir de su relación con el objetivo y el contenido de la investigación. La búsqueda en internet permitió seleccionar aquellos artículos que mejor explicaban las relaciones entre las categorías principales en estudio, es decir, formación investigativa, enfoque CTS en el ámbito educativo y Problemas Sociales de la Ciencia y la Tecnología. Los artículos se seleccionaron, además, porque cumplían el requisito de estar publicados en revistas certificadas e indexadas en bases de datos de nivel máximo y medio para los estudios de doctorados en Cuba, en el área de conocimientos de ciencias pedagógicas (Scielo, Redalyc, Dialnet Plus y ERIH Plus). Se adicionaron al estudio tres libros que permitieron la sistematización teórica sobre los fundamentos didácticos, la formación profesional y formación investigativa. Se incluyó un libro que aborda la metodología de la sistematización como metodología de investigación y uno que aborda la relación universidad-ciencia. A partir de este estudio se establecieron relaciones entre, la formación investigativa de los estudiantes universitarios, el enfoque CTS y el PEA de la asignatura PSCT.

Las relaciones reveladas permitieron elaborar indicadores para evaluar la formación investigativa de los estudiantes en el PEA de la asignatura PSCT que son los siguientes:

Indicador 1. Demuestra dominio en el procesamiento de la información sobre las revisiones bibliográficas relacionadas con el contenido de cada tema.

Indicador 2. Fundamenta la selección de un tema vinculado a la disciplina integradora, con enfoque CTS. Para el ejercicio final de la asignatura.

Indicador 3. Asume posiciones críticas al valorar las condicionantes y determinantes sociales contextualizados en el tema de su investigación.

Indicador 4. Fundamenta en el tema de su investigación las políticas públicas en desarrollo científico Tecnológico.

Se aplicó una encuesta a 20 profesores, de 15 años de experiencia como promedio, de la Universidad de Ciego de Ávila (15 profesores titulares y cinco auxiliares) para determinar las causas de las insuficiencias en la formación investigativa de los estudiantes. En la encuesta se entregó un listado de criterios para que los profesores la ordenaran por manifestaciones y causas y le dieran una jerarquía por grado de importancia.

Los indicadores fueron evaluados a través del producto de la actividad de los estudiantes en los diferentes talleres. El taller final permitió evaluar todos los indicadores. Los indicadores 1, 2 y 3 relacionados con el dominio en el procesamiento de la información, fundamentar la selección de un tema vinculado a la disciplina integradora y asumir posiciones críticas al valorar las condicionantes y determinantes sociales contextualizados en el tema de su investigación fueron evaluados en todos los cinco talleres.

El indicador 4. Fundamenta en el tema de su investigación las políticas públicas en desarrollo científico Tecnológico se evaluó en los talleres 3, 4 y cinco, porque es donde el estudiante está preparado para dar respuestas a la tarea investigativa, es por eso que no se evalúa desde el inicio. Método estadístico: la aplicación de una prueba no paramétrica Kruskal-Wallis para analizar la existencia de diferencias significativas en la transformación de los indicadores. La evaluación de estos indicadores está reflejada en las tablas 1, 2, 3, 4, 5 y 6. 


\section{Análisis e interpretación de los resultados.}

En la encuesta a los profesores se precisaron como las manifestaciones que predominan como insuficiencias en la formación investigativa de los estudiantes las siguientes:

- Insuficiente dominio de habilidades investigativas para procesar la información de las fuentes.

- Falta de motivación de los estudiantes por la investigación

- Comportamientos reproductivos en los análisis de contenido.

- Pobre participación del estudiantado en tareas de investigación.

- Insuficiente independencia cognitiva de los estudiantes.

Los resultados de la encuesta aplicada a los profesores indican que las causas que más inciden en las insuficiencias que se manifiestan en la formación investigativa de los estudiantes son:

- El proceso de enseñanza aprendizaje muy reproductivo, 18 de los 20 encuestados (90\%).

- Insuficiente preparación teórica metodológica de los profesores sobre la enseñanza problémica 18 de los 20 encuestados (90\%).

- Insuficiente orientación metodológica en los procesos investigativos a los estudiantes 16 de los 20 encuestados (80\%).

- En la enseñanza aprendizaje predomina el modelo explicativo ilustrativo 15 de los 20 encuestados $(75 \%)$.

Rasgos del proceso de la formación investigativa que se sintetizan a partir del estudio de contenido de los artículos y libros consultados:

- El proceso de formación investigativa exige de producción de conocimientos unificados por un campo conceptual común, organizado y regulados por un sistema de normas (Castell \& Ipola, 1975).

- La formación desde el punto de vista investigativo, puede impartirse a través de todas las actividades docentes (Restrepo- Gómez, 2003).

- Es un proceso de incorporación y participación en la cultura mediado por símbolos, rituales y relaciones sociales entre los cuales están las relaciones pedagógicas (RojasGranada y Aguirre-Cano, 2015).

- Proceso que implica práctica y actores diversos (Rojas-Granada y Aguirre-Cano, 2015).

- La investigación universitaria como un proceso de búsqueda de conocimientos (RestrepoGómez, 2003).

- Proceso que busca la generación de conocimientos donde se involucra no solo la comprensión del hombre mismo (Maldonado, Landazábal, Hernández, Ruiz, Claro, Venegas y Cruz, 2007).

- Se inicia el desarrollo de la cultura investigativa-ideológica crítica (Maldonado, Landazábal, Hernández, Ruiz, Claro, Venegas y Cruz, 2007).

- Los planes de estudios deben propiciar una cultura investigativa a través de la síntesis y retroalimentación entre la docencia y la investigación en el proceso de formación (González-Melo, 2011).

- En el proceso de formación se fomenta una postura investigativa que le posibilita al estudiante enfrentarse a su praxis en el contexto de aprendizaje (Hilarraza, 2012).

- Implica análisis crítico y reflexivo de sus estructuras investigativas (Hilarraza, 2012).

- El desarrollo de la cultura investigativa como proceso complejo a través del PEA (Aldana de Becerra, 2012).

- Intercambio grupal, auto preparación para la actividad docente (Márquez-Valdés y AcostaUrbano, 2014). 
Un estudio de contenido de la revisión de diferentes autores permitió sintetizar los rasgos de la formación investigativa de los estudiantes.

- Relación de la comprensión del mundo y la comprensión del hombre mismo (Maldonado, Landazábal, Hernández, Ruiz, Claro, Venegas y Cruz, 2007).

- Desarrollo de la cultura investigativa ideológicamente crítica y autónoma, que permite adherirse a los adelantos del conocimiento (Maldonado, Landazábal, Hernández, Ruiz, Claro, Venegas y Cruz, 2007); (González-Melo, 2011); (Aldana de Becerra, 2012).

- Empleo de recursos tecnológicos idóneos para el desarrollo de la investigación científica (Estrada-Hernández y Cristancho- Marulanda, 2014).

- Formación de la competencia investigativa sobre la base de las dimensiones de: saber (conocimiento), saber hacer (habilidades y destrezas), saber ser (aptitudes y actitudes) (Márquez-Valdés, y Acosta-Bandomo, 2014); (Maldonado, Landazábal, Hernández, Claro, Venegas, Cruz, 2007); (González-Melo (2011); (Pla-López, 2017); (Zamora-Vera, 2014) y (Zúñiga-García y Pando-Ezcurra, 2019).

- Fundamentación epistemológica y métodos científicos (Estrada-Hernández y CristanchoMarulanda, 2014) y (Pla-López,2017).

- Elaboración de proyectos (Estrada-Hernández y Cristancho- Marulanda, 2014) y (PlaLópez, 2017).

- Análisis crítico y reflexivo del estado del arte (Hilarraza, 2012).

- Posturas éticas y epistemológicas (Hilarraza, 2012).

- Análisis crítico del contexto lo cual puede llevar a procesos de autoevaluación y de articulación de la investigación formativa científica (Márquez-Valdés y Acosta Bandomo, 2014).

- Capacidad del estudiante para resolver independientemente los problemas a un nivel potencial con una guía o experto (Zamora-Vera, 2014) y Rojas-Granada y Aguirre-Cano, 2015).

Como se aprecia hay disímiles criterios, pero todos responden a que existe una necesidad de la formación desde el punto de vista investigativo, en la enseñanza universitaria, además reconocen que es un proceso complejo y se manifiestan diferentes vías para su realización, que se han obtenido resultados parciales y contextualizados. Reconocen que una de las vías es el propio proceso de enseñanza aprendizaje por su carácter organizacional, sistémico, donde intervienen diferentes actores, fundamentalmente el profesor y los estudiantes.

El estudio de contenido de los autores consultados permitió establecer un vínculo entre: Formación Investigativa- Enfoque CTS- Proceso Enseñanza Aprendizaje de la asignatura PSCT que se presenta a continuación:

Cuadro no. 1. Síntesis del estudio de contenido de categorías claves en la investigación.

\begin{tabular}{|c|c|c|}
\hline $\begin{array}{l}\text { Formación Investigativa } \\
\text { Como proceso. }\end{array}$ & Enfoque CTS. & PEA de la asignatura PSCT. \\
\hline $\begin{array}{l}\text { 1.-Producción de conocimientos } \\
\text { unificados por un campo conceptual } \\
\text { común, organizados y regulados por } \\
\text { un sistema de normas. } \\
\text { 2.- La formación desde el punto de } \\
\text { vista investigativo, puede impartirse a } \\
\text { través de otras actividades docentes. } \\
\text { 3.-Proceso de búsqueda de nuevos } \\
\text { con conocimientos. }\end{array}$ & $\begin{array}{l}\text { 1.-La enseñanza con } \\
\text { orientación CTS puede } \\
\text { ayudar a modificar la } \\
\text { praxis docente desde dos } \\
\text { puntos de vista } \\
\text { complementarios: el } \\
\text { papel del profesor y las } \\
\text { estrategias de enseñanza } \\
\text { aprendizaje. }\end{array}$ & $\begin{array}{l}\text { 1. Proceso organizado. } \\
\text { Responde a leyes, principios } \\
\text { y categorías de la didáctica } \\
\text { general. } \\
\text { 2.- Búsqueda de nuevos } \\
\text { conocimientos sobre } \\
\text { Ciencia y Tecnología. } \\
\text { 3. Caracteriza la Ciencia y la } \\
\text { Tecnología como procesos } \\
\text { sociales. }\end{array}$ \\
\hline
\end{tabular}




\begin{tabular}{|c|c|c|}
\hline $\begin{array}{l}\text { 4.- Caracterizado por la creación del } \\
\text { acto de innovación de ideas. } \\
\text { 5.- De incorporación y participación } \\
\text { en la cultura mediada por símbolos, } \\
\text { rituales y relaciones sociales entre las } \\
\text { cuales están las relaciones } \\
\text { pedagógicas. } \\
\text { 6.- Implica práctica y actores } \\
\text { diversos. } \\
\text { 7.-Proceso que busca la generación } \\
\text { de conocimientos, donde se } \\
\text { involucra no sólo la comprensión del } \\
\text { hombre mismo. } \\
\text { 8.- Se inicia de alguna manera el } \\
\text { desarrollo de la cultura investigativa } \\
\text { ideológica y crítica. } \\
\text { 9.-Los planes de estudios deberían } \\
\text { propiciar la investigación de } \\
\text { formación generando una cultura } \\
\text { investigativa a través de la síntesis y } \\
\text { retroalimentación entre la docencia y } \\
\text { la investigación fon postura } \\
\text { 10.- Se fomenta una postura } \\
\text { investigativa que le posibilita al } \\
\text { estudiante enfrentar a su praxis en el } \\
\text { contexto de aprendizaje } \\
\text { 11.- Implica análisis crítico y reflexivo } \\
\text { de sus estrategias investigativas. } \\
\text { 12.-Cultura investigativa como } \\
\text { proceso complejo atinente a una } \\
\text { cultura organizacional del PEA. } \\
\text { 13.-Intercambio grupal } \\
\text { autopercepción. }\end{array}$ & $\begin{array}{l}\text { 2.-Principio central es la } \\
\text { contextualización. } \\
\text { 3.-Los sistemas } \\
\text { educativos deben } \\
\text { esforzarse por saber algo } \\
\text { sobre la ciencia, en } \\
\text { especial respecto a sus } \\
\text { características culturales, } \\
\text { sus epistemológicos, los } \\
\text { conceptos éticos que la } \\
\text { envuelven y su } \\
\text { interacción con la } \\
\text { sociedad. pa de entender } \\
\text { 4.- Se ha de preparación } \\
\text { como una prón } \\
\text { para la participación } \\
\text { ciudadana, siendo estas } \\
\text { metas propias de la } \\
\text { educación desde una } \\
\text { perspectiva CTS } \\
\text { 5.-Potencia los valores de } \\
\text { la Ciencia y la Tecnología } \\
\text { para poder entender } \\
\text { mejor lo que éstas } \\
\text { pueden aportar. } \\
\text { 6.- Es un campo } \\
\text { multidisciplinario } \\
\text { interdisciplinario con una } \\
\text { explícita voluntad de } \\
\text { superar las fronteras que } \\
\text { frecuentemente cuartean } \\
\text { los currículos. } \\
\text { 7.- Muestra que la } \\
\text { Ciencia y la Tecnología } \\
\text { son construcciones } \\
\text { humanas y que, por } \\
\text { tanto, reflejan los deseos, } \\
\text { intereses y valores de los } \\
\text { humanos. }\end{array}$ & $\begin{array}{l}\text { 4.-Enseñanza dialogada, } \\
\text { participativa. } \\
\text { 5.-Requiere lograr una } \\
\text { enseñanza con enfoque } \\
\text { CTS, por sus objetivos. } \\
\text { 6.-Los estudiantes asumen } \\
\text { criterios para enjuiciar, } \\
\text { valorar problemas } \\
\text { relacionados con la Ciencia y } \\
\text { la Tecnología. } \\
\text { 7.- El profesor utiliza } \\
\text { métodos y medios que } \\
\text { promueve la posibilidad de } \\
\text { la participación de los } \\
\text { estudiantes. } \\
\text { 8.-Adquiere una cultura } \\
\text { investigativa para la gestión } \\
\text { del aprendizaje. } \\
\text { 9.-El estudiante demuestra } \\
\text { la solución de problemas de } \\
\text { su profesión con enfoque } \\
\text { CTS. } \\
\text { 10.- Se organiza el PEA a } \\
\text { través de distintas formas } \\
\text { organizativas. } \\
\text { 11.-Predominan } \\
\text { métodos de la enseñanza } \\
\text { problémica. }\end{array}$ \\
\hline
\end{tabular}

Fuente: Elaboración de los autores a partir del estudio de contenidos.

El estudio de los rasgos de la formación investigativa de los estudiantes y su relación con el enfoque CTS permitió establecer procederes didácticos para el perfeccionamiento en el PEA de la asignatura PSCT.

\section{Proceder didáctico del PEA de la asignatura PSCT para la formación investigativa.}

1.Debe procederse con intencionalidad hacia la formación desde el punto de vista investigativo. Está dada por la formulación de los objetivos a nivel de clase y tarea docente. Esa intención se propone en todas las tareas docentes relacionadas con el componente investigativo. RamosBañobre y Pla-López (2016) consideran, que la investigación es una actividad cognoscitiva 
compleja que revela en forma de proceso, cuyas características esenciales son la sistematicidad, intencionalidad, necesaria de su planificación y la orientación hacia la búsqueda de nuevos conocimientos que resuelvan un problema.

2.La motivación es una exigencia en la orientación del nuevo contenido y en todo el proceso. Si el estudiante no se siente motivado no reafirma los conocimientos. Esta motivación tiene que establecerse con el perfil profesional y los problemas ingenieriles que despierte el interés de los estudiantes.

3. Contextualización como proceder del profesor y del estudiante. Se fomenta una postura investigativa que posibilita al estudiante enfrentarse a su praxis en el contexto de aprendizaje. Además, se fortalecen valores identitarios que fortalecen la formación profesional.

4. Estudiar la ciencia y la tecnología como construcciones humanas y con implicaciones sociales. Este requerimiento es además uno de los objetivos generales de la asignatura.

5. Enfoque crítico e interdisciplinario. La enseñanza con enfoque CTS requiere de una enseñanza dialogada, problémica donde el estudiante participe, da criterios cuyos fundamentos están en la búsqueda realizada, en los contenidos con otras asignaturas. La formación desde el punto de vista investigativo requiere de una enseñanza problémica que promueva el pensamiento de los estudiantes a la búsqueda, planteamientos y solución de problemas.

6.El uso de los medios de enseñanza, preferentemente audiovisuales, que permita visualización de aspectos relacionados con la ciencia y la tecnología que son muy difícil de explicar solo con la palabra oral o escrita. Esto proporciona objetividad al proceso.

7.Las formas organizativas tienen que proporcionar el debate, la participación por lo que se deben establecer clases prácticas, seminarios, talleres (predominan) y las conferencias orientadoras.

8.La evaluación como parte del proceso tiene que ser sistemática y orientarse en cada momento para el resultado final.

9. Revelar el enfoque CTS en cada uno de los temas de la asignatura.

10. Tareas docentes dirigidas a la investigación sobre temas de la especialidad en vínculo con la disciplina integradora del año.

Estos procederes didácticos elaborados se utilizaron para perfeccionar el PEA de la asignatura PSCT, en el tercer año de la carrera de Ingeniería Civil, durante el curso 2019-2020, para contribuir a la formación investigativa de los estudiantes.

\section{Transformación de la formación investigativa de los estudiantes.}

Los datos se obtienen a partir del registro de cuatro evaluaciones sistemáticas durante el PEA de la asignatura PSCT y un taller final en el que presentaron trabajos investigativos con temas vinculados al ejercicio de la profesión.

Se utilizó una escala de 2 a 5 puntos, para medir los resultados, en la que cinco (5) es la calificación máxima y dos (2) es la mínima. Se evaluaron los cuatro indicadores operacionalizados en la metodología.

Tabla No 1. Resultados de la evaluación 1

\begin{tabular}{|l|l|l|l|l|l|}
\hline $\begin{array}{l}\text { Evaluación } \\
1\end{array}$ & $\begin{array}{l}\text { Total, de } \\
\text { alumnos } \\
\text { evaluados }\end{array}$ & $\begin{array}{l}\text { Alumnos } \\
\text { con 2 } \\
\text { puntos }\end{array}$ & $\begin{array}{l}\text { Alumnos } \\
\text { con } \\
\text { puntos }\end{array}$ & $\begin{array}{l}\text { Alumnos } \\
\text { con } \\
\text { puntos }\end{array}$ & $\begin{array}{l}\text { Alumnos } \\
\text { con 5 } \\
\text { puntos }\end{array}$ \\
\hline Indicador 1 & 16 & 10 & 6 & 0 & 0 \\
\hline Indicador 2 & 16 & 9 & 7 & 0 & 0 \\
\hline Indicador 3 & 16 & 8 & 8 & 0 & 0 \\
\hline
\end{tabular}

Fuente: Elaboración propia.

Tabla No 2. Resultados de la evaluación 2 


\begin{tabular}{|l|l|l|l|l|l|}
\hline $\begin{array}{l}\text { Evaluación } \\
2\end{array}$ & $\begin{array}{l}\text { Total, de } \\
\text { alumnos } \\
\text { evaluados }\end{array}$ & $\begin{array}{l}\text { Alumnos } \\
\text { con 2 } \\
\text { puntos }\end{array}$ & $\begin{array}{l}\text { Alumnos } \\
\text { con } \\
\text { puntos }\end{array}$ & $\begin{array}{l}\text { Alumnos } \\
\text { con } \\
\text { puntos }\end{array}$ & $\begin{array}{l}\text { Alumnos } \\
\text { con 5 } \\
\text { puntos }\end{array}$ \\
\hline Indicador 1 & 16 & 0 & 13 & 3 & 0 \\
\hline Indicador 2 & 16 & 5 & 9 & 2 & 0 \\
\hline Indicador 3 & 16 & 0 & 12 & 3 & 1 \\
\hline
\end{tabular}

Fuente: Elaboración propia.

Nota aclaratoria: Se incorpora a partir de este taller el indicador 4 pues requiere de los conocimientos adquiridos por los estudiantes.

Tabla No 3. Resultados de la evaluación 3

\begin{tabular}{|l|l|l|l|l|l|}
\hline $\begin{array}{l}\text { Evaluación } \\
3\end{array}$ & $\begin{array}{l}\text { Total, de } \\
\text { alumnos } \\
\text { evaluados }\end{array}$ & $\begin{array}{l}\text { Alumnos } \\
\text { con 2 } \\
\text { puntos }\end{array}$ & $\begin{array}{l}\text { Alumnos } \\
\text { con } \\
\text { puntos }\end{array}$ & $\begin{array}{l}\text { Alumnos } \\
\text { con } \\
\text { puntos }\end{array}$ & $\begin{array}{l}\text { Alumnos } \\
\text { con 5 } \\
\text { puntos }\end{array}$ \\
\hline Indicador 1 & 16 & 0 & 13 & 3 & 0 \\
\hline Indicador 2 & 16 & 0 & 9 & 7 & 0 \\
\hline Indicador 3 & 16 & 0 & 10 & 6 & 0 \\
\hline Indicador 4 & 16 & 5 & 9 & 2 & 0 \\
\hline
\end{tabular}

Fuente: Elaboración propia.

Tabla No 4. Resultados de la evaluación 4

\begin{tabular}{|l|l|l|l|l|l|}
\hline $\begin{array}{l}\text { Evaluación } \\
4\end{array}$ & $\begin{array}{l}\text { Total, de } \\
\text { alumnos } \\
\text { evaluados }\end{array}$ & $\begin{array}{l}\text { Alumnos } \\
\text { con 2 } \\
\text { puntos }\end{array}$ & $\begin{array}{l}\text { Alumnos } \\
\text { con } \\
\text { puntos }\end{array}$ & $\begin{array}{l}\text { Alumnos } \\
\text { con } \\
\text { puntos }\end{array}$ & $\begin{array}{l}\text { Alumnos } \\
\text { con 5 } \\
\text { puntos }\end{array}$ \\
\hline Indicador 1 & 16 & 0 & 11 & 5 & 0 \\
\hline Indicador 2 & 16 & 0 & 2 & 12 & 2 \\
\hline Indicador 3 & 16 & 0 & 2 & 10 & 4 \\
\hline Indicador 4 & 16 & 0 & 0 & 11 & 5 \\
\hline
\end{tabular}

Fuente: Elaboración propia.

Tabla No 5. Resultados de la evaluación del Taller Final

\begin{tabular}{|l|l|l|l|l|l|}
\hline Taller Final & $\begin{array}{l}\text { Total, de } \\
\text { alumnos } \\
\text { evaluados }\end{array}$ & $\begin{array}{l}\text { Alumnos } \\
\text { con 2 } \\
\text { puntos }\end{array}$ & $\begin{array}{l}\text { Alumnos } \\
\text { con } \\
\text { puntos }\end{array}$ & $\begin{array}{l}\text { Alumnos } \\
\text { con } \\
\text { puntos }\end{array}$ & $\begin{array}{l}\text { Alumnos } \\
\text { con 5 } \\
\text { puntos }\end{array}$ \\
\hline Indicador 1 & 16 & 0 & 0 & 5 & 11 \\
\hline Indicador 2 & 16 & 0 & 0 & 5 & 11 \\
\hline Indicador 3 & 16 & 0 & 0 & 2 & 14 \\
\hline Indicador 4 & 16 & 0 & 0 & 2 & 14 \\
\hline
\end{tabular}

Fuente: Elaboración propia. (2020).

Los resultados expresados en las tablas 1, 2, 3, 4 y 5 permiten comprender que hubo un proceso de desarrollo cuanti-cualitativos en los indicadores evaluados. Avanzando hacia evaluaciones máximas en las últimas mediciones.

Se aplicó la prueba no paramétrica Kruskal-Wallis para evaluar las transformaciones de los indicadores en diferentes momentos (talleres) con una significación de 0,05 , para comprobar la existencia de diferencias significativas. 
Tabla No 6. Tests Kruskal-Wallis Test

Test Statistics ${ }^{\mathrm{a}, \mathrm{b}}$

\begin{tabular}{|l|l|}
\hline & Indicador1 \\
\hline Chi-Square & 51,366 \\
Df & 4 \\
Asymp. Sig. & 000 \\
\hline & indicador2 \\
\hline Chi-Square & 39,276 \\
Df & 4 \\
Asymp. Sig. &, 000 \\
\hline & Indicador3 \\
\hline Chi-Square & 46,582 \\
Df & 4 \\
Asymp. Sig. &, 000 \\
\hline \hline & Indicador4 \\
\hline Chi-Square & 49,126 \\
Df & 2 \\
Asymp. Sig. &, 000 \\
\hline
\end{tabular}

Fuente: Procesamiento estadístico SPSS.V 23

Los resultados de la aplicación de la prueba no paramétrica Kruskal-Wallis reflejan la existencia de diferencia significativas en las transformaciones de los 4 indicadores evaluados en talleres mostrando en cada evaluación una Asymp. Sig. 0.00 menor que 0,05 .

Al finalizar los temas de la asignatura en el primer semestre del curso 2019-2020 se realizó un Taller integrador en el que los estudiantes organizados por equipos demostraron, tener dominio del contenido de las categorías fundamentales del programa de la asignatura PSCT, en cuanto al enfoque Ciencia, Tecnología y Sociedad (CTS); lograron presentar sus trabajos finales con rigor científico en el tratamiento de la información de los autores consultados; sus trabajos reflejan coherencia entre problema científico, objetivo, conclusiones; existió calidad de las ideas y redacción; los temas tienen una relación con el perfil profesional y relacionado con los proyectos de la disciplina integradora del año y además demostraron valores identitario y posiciones críticas en cuanto a las condicionantes, determinantes sociales y las políticas públicas, en los temas presentados.

\section{Discusión}

Los resultados teóricos y prácticos mostraron coincidencias con los criterios de los autores estudiados sobre la formación investigativa.

La formación desde el punto de vista investigativo de los estudiantes universitarios puede impartirse a través de las actividades docentes desde cada asignatura, lo que confirma los criterios asumidos de Restrepo-Gómez (2003).

En el proceso investigativo se pudo constatar el criterio asumido de Rojas y Aguirre (2015) de que el proceso de formación investigativa de los estudiantes implica práctica sistemática y la presencia de actores diversos, ya que es necesario incorporar a personas de la comunidad, sobre todo, a trabajadores que tienen que ver con los procesos constructivos de la región.

Fue muy significativo en el proceso investigativo el criterio de Maldonado, Landazábal, Hernández, Ruiz, Venegas, Cruz (2007), que enfatiza que el proceso de formación desde el punto de vista investigativo genera en el estudiante la comprensión de sí mismo y de su papel en la sociedad lo que inicia el desarrollo de la cultura investigativa-ideológica y crítica del estudiante. 
Para los autores de la investigación quedó revelado que el PEA de las asignaturas del currículo de estudios deben propiciar una cultura investigativa a través de la síntesis y retroalimentación entre la docencia y la investigación en el proceso de formación, lo que coincide con el criterio de GonzálezMelo (2011). Por otra parte, el en proceso de formación se fomenta una postura investigativa que le posibilita al estudiante enfrentarse a su praxis en el contexto de aprendizaje, lo que reafirma el principio de contextualización del enfoque CTS en el ámbito educativo defendido por Hilarraza (2012).

El PEA de la asignatura para contribuir a la formación investigativa de los estudiantes debe asumir un proceder didáctico que implique el análisis crítico y reflexivo de sus estructuras investigativas, es por eso que los estudiantes deben enfrentarse en las actividades que desarrollan, a la selección de temas relacionados con su profesión, procesar información de las fuentes con rigor científico, determinar problemas para resolverlos a través del método científico y sistematizar la propuesta de soluciones, todo en relación con los problemas profesionales de la ingeniería civil. Estos rasgos de la formación investigativa de los estudiantes, son reflejados por la mayoría de los autores estudiados: Estrada-Hernández y Cristancho- Marulanda (2014); Márquez-Valdés y Acosta-Bandomo (2014); Maldonado, Landazábal, Hernández, Claro, Venegas, Cruz (2007); González-Melo (2011), PlaLópez (2017); Zamora-Vera (2014) y Zúñiga-García y Pando-Ezcurra (2019).

\section{Conclusiones}

Los procederes didácticos sistematizados y aplicados en el proceso de enseñanza aprendizaje de la asignatura Problemas Sociales de la Ciencia y la Tecnología contribuyen, como los resultados indican, a la formación investigativa de los estudiantes universitarios de la carrera de Ingeniería Civil, lo cual es posible, si se parte de la relación con los problemas profesionales, para la contextualización de los diferentes temas. Esta característica refuerza la motivación por el estudio de la asignatura y mayor calidad de su formación profesional.

Los estudios teóricos realizados permiten revelar relaciones que se dan entre la formación investigativa de los estudiantes universitarios de la carrera de Ingeniería Civil y el enfoque CTS en el ámbito educativo que se ha venido desarrollando en el mundo en las últimas décadas. Esas relaciones cuando se tienen en cuenta en el diseño, desarrollo y evaluación del proceso de enseñanza aprendizaje de la asignatura Problemas Sociales de la Ciencia y la Tecnología permiten perfeccionar el proceder didáctico que se asume por estudiantes y profesores en dicho proceso.

\section{Referencias Bibliograficas}

Acevedo-Díaz, José Antonio. (1997). Ciencia, Tecnología y Sociedad. Un enfoque innovador para la Enseñanza de las Ciencias. Revista de Educación de la Universidad de Granada. Volumen 1, N. ${ }^{\circ} \quad 10, \quad$ España $\quad$ (Pp. 269-275). https://dialnet.unirioja.es/servlet/articulo?codigo $=119565$

Aldana de Becerra, Gloria Marlen. (2012). La formación investigativa. Su pertinencia en el pregrado. Revista Virtual Universidad Católica del Norte. Volumen 35, No (1), Colombia (Pp. 367-379). https://www.redalyc.org/pdf/1942/194224362019.pdf

Álvarez de Zayas, Carlos Manuel. (1990). Elementos de la didáctica de la Educación Superior. Editora M.E.S. Cuba.

Castell, Manuel \& Ipola, Emilio. (1975). Metodología y Epistemología de las Ciencias Sociales. Editorial Ayuso, España.

Estrada Hernández, Margarita y Cristancho Marulanda, Sergio (2014) La información científica en la investigación sobre salud Mental en la Universidad de Antioquía. Revista Cubana de Información de Ciencias de la salud. Volumen 25, No. (1). Cuba. (Pp.4-23) https://www.researchgate.net/publication/261295782 La informacioncientifica en la in vestigacion sobre salud mental en la Universidad de Antioquia Colombia 
González-Melo, Hamlet Santiago. (2011). Formación Investigativa para la Educación Superior desde una Perspectiva pedagógica. Revista Científica, Volumen 14, N. 2. Colombia (Pp.7278). https://revistas.udistrital.edu.co/index.php/revcie/article/view/3702

Hilarraza, Yaritza Josefina. (2012). La investigación pedagógica: un aporte para a la gestión de la formación docente desde un punto de vista socio cultural. Revista Didasc@lia: Didáctica y Educación. Volumen 3, No. 1, Cuba. (Pp.25-40) https://dialnet.unirioja.es/servlet/articulo?codigo $=4228367$

Maldonado, Luis Facundo; Landazábal, Diana Patricia; Hernández, Juan Carlos; Ruiz Yasbleidy; Audrey Claro; Venegas, Harvert; Cruz Sandra (2007). Visibilidad y formación investigativa. Estrategias para el desarrollo de competencias investigativas. Studiositas. Volumen 2. No. (2). Colombia (Pp.43-56). Recuperado: http://repository.ucatolica.edu.co/handle/10983/502

Márquez Valdés, Annia María y Acosta Bandomo, Rafael Urbano (2014). La formación de competencias investigativas en estudiantes de Cultura Física con un enfoque CTS. EFDeportes.com, Revista Digital. Buenos Aires, Volumen 19, No.197, Argentina. (Pp.1-14). Recuperado: https://www.efdeportes.com/efd197/la-formacion-decompetencias-investigativas.htm

Núñez Jover, Jorge. (2019). Universidad, Conocimiento y desarrollo: nuevas encrucijadas. Una lectura desde Ciencia, Tecnología y Sociedad. Editorial U. H. (Universidad de La Habana). Cuba.

Osorio, Carlos. (2002). La educación científica y tecnológica desde un enfoque Ciencia, Tecnología y Sociedad. Revista Iberoamericana de Educación. No 28, México (pp. 61-81). https://www.redalyc.org/pdf/800/80002803.pdf

Pla López, Ramón. (2017). Modelo del profesional de la Educación: Sus competencias docentes. Editorial Academia Española. España

Ramos Bañobre, José María y Pla López, Ramón Vidal. (2016). La sistematización como metodología y método y resultado Científico investigativo en la práctica en educación. Editorial Ibarra, Ecuador.

Restrepo Gómez, Bernardo (2003). Aportes de la investigación acción educativa a la hipótesis del maestro investigador. Revista Educación y Educadores. N. 6, Colombia (Pp.91-104) Recuperado: http://www.redalyc.org/articulo.oa?id=83400607

Rojas Granada, Cristian y Aguirre Cano, Sebastián (2015). La formación investigativa en la educación superior en América Latina y el Caribe: una aproximación a su estado del arte. Revista Eleuthera. Volumen 12, Colombia (Pp.197-222). https://doi.org/10.17151/eleu.2015.12.11

Vilches, Amparo; Gil, Daniel; Cañal de León, Pedro. (2010). Educación para la sostenibilidad y educación ambiental. Revista Investigación en la Escuela. No 71 España (Pp.5-15) https://idus.us.es/handle/11441/60197

Zamora Vera, Narciza. (2014). La formación investigativa de los estudiantes: un problema por resolver. Tesis Doctoral. Revista Escenarios. Volumen. 12, N. 2. Ecuador. (Pp.76-85). https://doi.org/10.15665/esc.v12i2.316

Zúñiga García, Ximena Jeanneth y Pando Ezcurra, Tamara Tatiana. (2019). Las competencias investigativas y el aprendizaje basado en problemas. Un estudio cuasiexperimental. Revista Dilemas contemporáneos: Educación, Política y valores. Volumen 6, No. 2. México (Pp.1-23).

https://www.dilemascontemporaneoseducacionpoliticayvalores.com/index.php/dilemas/ar ticle/view/945/935 